\title{
Lateral Cerebellar Hemispheres Actively Support Sensory Acquisition and Discrimination Rather Than Motor Control
} Lawrence M. Parsons, ${ }^{1,3}$ James M. Bower, ${ }^{2}$ Jia-Hong Gao, ${ }^{1}$ Jinhu Xiong, ${ }^{1}$ Jinqi Li, ${ }^{1}$ and Peter T. Fox ${ }^{1}$

${ }^{1}$ Research Imaging Center

The Medical School

The University of Texas Health Science Center at San Antonio

San Antonio, Texas 78284

${ }^{2}$ Computation and Neural Systems

California Institute of Technology

Pasadena, California 91125

\section{Abstract}

This study examined a new hypothesis proposing that the lateral cerebellum is not activated by motor control per se, as widely assumed, but is engaged during the acquisition and discrimination of tactile sensory information. This proposal derives from neurobiological studies of these regions of the rat cerebellum. Magnetic resonance imaging of the lateral cerebellar output nucleus (dentate) of humans during passive and active sensory tasks confirmed four a priori implications of this hypothesis. Dentate nuclei responded to cutaneous stimuli, even when there were no accompanying overt finger movements. Finger movements not associated with tactile sensory discrimination produced no dentate activation. Sensory discrimination with the fingers induced an increase in dentate activation, with or without finger movements. Finally, dentate activity was greatest when there was the most opportunity to modulate the acquisition of the sensory tactile data: when the discrimination involved the active repositioning of tactile sensory surface of the fingers. Furthermore, activity in cerebellar cortex was strongly correlated with observed dentate activity. This distinct

${ }^{3}$ Corresponding author. four-way pattern of effects strongly challenges other cerebellar theories. However, contrary to appearances, neither our hypothesis nor findings conflict with behavioral effects of cerebellar damage, neurophysiological data on animals performing motor tasks, or cerebellar contribution to nonmotor, perceptual, and cognitive tasks.

\section{Introduction}

The cerebellum has been viewed by most neurologists and neurobiologists in this century as principally a motor organ (Holmes 1917; Thach et al. 1992; Glickstein 1993; Ito 1993; Paulin 1993; Courchesne et al. 1994; Daum and Ackermann 1995; Dean 1995; Filapek 1995; Houk and Wise 1995; Welsh et al. 1995; Thach 1997). The most conspicuous and therefore best-studied effects of cerebellar lesions are on movement coordination (Holmes 1939; Thach et al. 1992; Subramony 1994; Gilman et al. 1996). Classic theory has emphasized that the output of the deep cerebellar nuclei in mammals is to primarily influence motor structures (Asanuma et al. 1983; Aumann et al. 1994; Keifer and Houk 1994). Cerebellar activity during movement has been well confirmed by electrophysiological recordings in animals (Ojakangas and $\mathrm{Eb}$ ner 1992, 1994; Keifer and Houk 1994; van Kan et al. 1994; Apps et al. 1995) and by functional neuroimaging in humans (Fox et al. 1985; Ellerman et al. 1994). As a consequence, nearly all cellular and

LEARNING \& MEMORY 4:49-62 @ 1997 by Cold Spring Harbor Laboratory Press ISSN1072-0502/97 \$5.00

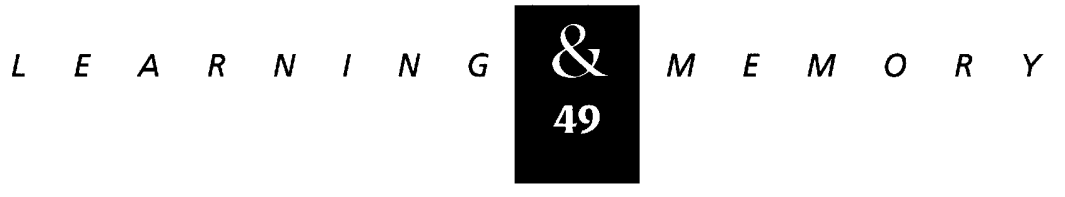


network level theories of cerebellar function focus on movement control (Marr 1969; Llinás 1984; Bloedel and Kelly 1992; Kawato and Gomi 1992; Paulin 1993; Houk and Wise 1995; Martin and Albers 1995). Despite the wealth of data concerning the possible involvement of the cerebellum in motor control, the specific computation executed by the cerebellum is far from established. For example, the role of the cerebellum in motor learning is a hotly contested issue, as demonstrated by the discussion in the present journal.

Be that as it may, the speculations on cerebellar function have recently been greatly diversified by numerous neurological, neuroimaging, and anatomical findings. Studies of brain-injured humans suggest that the cerebellum may be instrumental in nonmotor behaviors such as judging the timing of events, solving perceptual and spatial reasoning problems, and generating words according to a semantic rule (Bracke-Tolkmitt et al. 1989; Ivry and Keele 1989; Fiez et al. 1992; Grafman et al. 1992; Courchesne et al. 1994; Horak and Diener 1994). Support for some involvement in such perceptual and cognitive behaviors has also been obtained from recent neuroimaging studies of healthy humans (Petersen et al. 1989; Kim et al. 1994; Bower 1995; Jueptner et al. 1995; Leiner et al. 1995; Parsons et al. 1995). Furthermore, strong focused cerebellar activation has been detected for the mental rotation of abstract objects (L.M. Parsons and P.T. Fox, unpubl.), for different kinds of musical performance (P.T. Fox, L.M. Parsons, D.A. Hodges, and J.S. Sergent, in prep.), and for a variety of visual information processing tasks (Allen et al. 1997; Shulman et al. 1997). In these mental rotation, music, and visual studies, either motor behavior was not present in the task or activity owing to the task's motor components was dissociated from that owing to its nonmotor components. In addition, these functional data are consistent with recent anatomical studies that have discovered connectivity between cerebellum, midbrain, and nonmotor regions of the cerebral cortex (for recent discussion, see Middleton and Strick 1994; Schmahmann 1996, 1997).

Such findings clearly challenge classical motor theories of cerebellar function and have created an environment in which researchers are increasingly questioning the extent to which the cerebellum is involved in motor control. Although in most cases new alternative proposals are described as an extension of the role of the cerebellum in motor coordination itself, driven primarily by new neuroim- aging results, others are proposing radically different functions for this ancient structure (for reviews, see Doyon 1997; Fiez and Raichle 1997; Parsons and Fox 1997).

This paper tests one such new theory of cerebellar function that posits that the cerebellum is specifically involved in monitoring and adjusting the acquisition of most of the sensory data on which the rest of the nervous system depends. Although space does not permit a complete presentation of the arguments for this hypothesis (Bower 1992, 1997a,b), we note briefly that it was suggested by results from electrophysiological studies of the tactilely responsive regions of the rat cerebellum (Bower and Woolston 1983; Paulin et al. 1989b; Sasaki et al. 1989; Bower and Kassel 1990; Gonzalez et al. 1993; Jaeger and Bower 1994). These regions of the cerebellum have extensive tactile representations in rats but no reported representation from proprioceptors (Welker 1987). Furthermore, these tactile representations are dominated by representations of the lip and whisker regions of the rat's face rather than by representations of the fore- or hindlimb, as would be expected from an involvement in limb movement coordination. Only selected regions of the rat's face are represented in these maps: those regions on the "leading edge of the rat" that are known to be in direct contact with objects during sensory exploration (Jacquin and Zeigler 1983). Furthermore, there is an unusual fractured somatotopic pattern of the tactile projections that seems to assure that data from the different regions of the rat's face involved in sensory exploration are juxtaposed in different combinations, perhaps reflecting behavioral relationships between the different body surfaces involved in tactile exploration (Jacquin and Zeigler 1983). Finally, a reanalysis of the physiological organization of cerebellar cortical circuitry has suggested that this network may be organized very differently than heretofore assumed (Jaeger and Bower 1994). This new understanding of cerebellar cortical organization is consistent with the idea that these regions of cerebellum control the use of these different surfaces during tactile sensory data acquisition (Bower 1997a).

Classical cerebellar theory, of course, has assumed that the cerebellum does not have sensory functions. There are other grounds for reconsidering this assumption however. The cerebellum receives input from virtually every sensory system (Brodal 1978; Brodal 1981; Welker 1987), including vestibular (Naito et al. 1995), proprioceptive

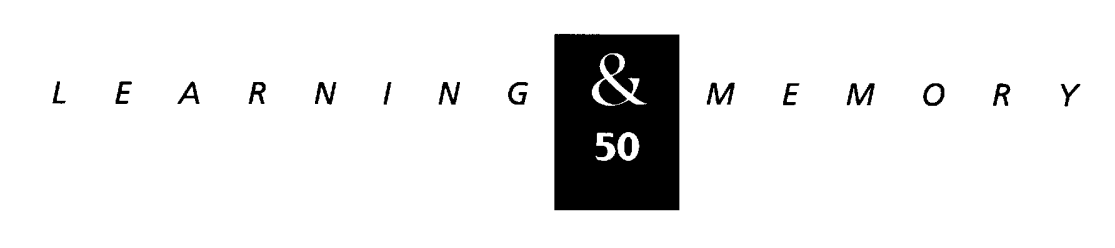


(Donga and Dessem 1993), visual (Snider and Stowell 1944), audition (Snider and Stowell 1944; Yang and Weisz 1992), somatosensation (Snider and Stowell 1944; Welker 1987), visceral pain receptors (Rubia and Phelps 1970), and sense pressure in blood vesicles (Nisimaru and Katayama 1995; Ghelarducci and Sebastiani 1996). In addition, it is activated by tactile stimulation alone (without overt movement) (Fox et al. 1985). Finally, determining whether a brain area has a motor or sensory function is a subtle, and often overlooked, problem. Motor behavior is guided by ongoing sensory acquisition of information about the object toward which action is directed. Continuously updated sensory data is necessary for accurate, coordinated, and smooth motor behavior. Nonetheless, we are convinced that it is necessary for motor operations to be disentangled as much as possible from sensory ones when testing hypotheses about cerebellar function.

Our study was designed specifically to dissociate tactile sensory data acquisition and discrimination from motor performance per se, by imaging blood flow change, a correlate of neural activity, in the lateral (dentate) nucleus of humans performing tasks both involving passive and active tactile discriminations. The dentate nucleus in humans is the sole output for the large lateral hemispheres of the primate cerebellum that are analogous to the region of tactile representation in the rat cerebellum (Larsell 1972) discussed above. In previous experimental studies of these regions in nonhuman primates, which are also known to respond to tactile stimuli, dentate and lateral hemisphere activation has been interpreted in the context of the control of finger movements for their own sake (Thach 1970, 1978; Strick 1983; van Kan et al. 1993). In an experimental study of this region in humans, Kim et al. (1994) used fMRI (functional magnetic resonance imaging) to assess activation in dentate nuclei during two tasks that were assumed to have comparable motor activity but different extents of "cognitive" processing (i.e., problem solving). The three times greater dentate activity detected during the more cognitive task was assumed to be attributable to nonmotor cognitive processing. However, this interpretation should be viewed with caution (Parsons and Fox 1997) because (1) only the more cognitive task was likely to involve imagined motor behavior and (2) imagined motor behavior is known to activate cerebellum as well as virtually all of the motor areas in cerebral cortex (Parsons et al. 1995).
In our study, we tested four implications of the alternative hypothesis that dentate activation will be more closely associated with sensory discriminations using the fingers than with finger-movement control in general. (1) Dentate nuclei should respond to sensory stimuli even when there are no accompanying overt finger movements. (2) Finger movements not associated with tactile sensory discrimination should not induce substantial dentate activation. (3) The requirement to make a sensory discrimination with the fingers should induce an increase in dentate activation, with or without accompanying finger movements. (4) The dentate should be most strongly activated when there is the most opportunity to modulate the acquisition of the sensory data, that is, when the sensory discrimination involves the active repositioning of tactile sensory surfaces through finger movements.

\section{Materials and Methods}

Six healthy volunteers performed four tasks. Subjects for the Cutaneous Stimulation (CS) and Cutaneous Discrimination (CD) tasks were males, ages 32-44 years (mean, 38). Five of those subjects and a female did the Grasped Objects (GO) and Grasped Objects Discrimination (GOD) tasks. All subjects gave informed consent. Subjects were given intensive practice session for 10 mins. In one session, they performed three cycles of Rest Control, CS, CD and, in another, three cycles of Rest Control, GO, GOD. Participants lay supine in a 1.9-T MRI instrument during each task. Subjects were blindfolded during all tasks and never saw the experimental stimuli.

In the CS Task, subjects passively experienced sandpaper rubbed against the immobilized pads of the second, third, and fourth fingers of each hand. In the CD Task, they were asked to actively compare (without responding) whether the coarseness of the sandpaper on their two hands matched. The coarseness of the sandpaper changed randomly every $3 \mathrm{sec}$. In the CS and CD tasks, movements were prevented by immobilization and instruction. The arm was immobilized by straps encircling the body. The wrist, hand, and fingers were immobilized by a rigid wooden surface affixed to the dorsum of the hand by tape encircling the wrist and fingers. During stimulation, subjects were instructed to allow their hand to remain flaccidly immobile, resting in the restraints. Sandpaper was applied to the finger pads by a continuous oscillation uncoordinated between hands. Four grades of

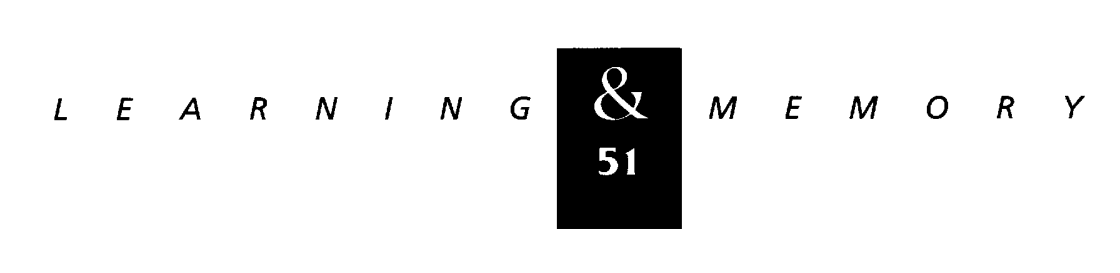


sandpaper were used: $60,100,150$, or 400 (U.S.A. Standard Grading system paper maximally packing grains of sand of $268,141,33$, and $23.6 \mu \mathrm{m}$ ). As the sandpaper was mildly aversive, involuntary movements toward the stimuli were unlikely. Involuntary movements away from the stimuli were prevented by the rigid surface to which the fingers were attached. Subjects were visually monitored for movement throughout each trial. No movements were observed.

In the two grasping tasks, each (unrestrained) hand was enclosed in its own tightly woven cotton sock, each sock containing an identical set of four differently shaped stimuli. Each stimulus was a 2.5mm-diameter wooden, smoothly surfaced sphere; the stimuli were differentiated by one-, two-, or three-faceted surfaces. In the GO Task, subjects were trained to pick up, roll in their fingers, and then release individual spheres with both sets of fingers. These instructions were intended to replicate movements made in the sensory discrimination as closely as possible, without a sensory discrimination actually being made. Subjects selected one of the four objects at random to grasp in each grasp cycle. This sequence was repeated continuously and was performed independently by each hand. In the GOD Task, subjects pincer-grasped a stimulus with one hand, felt its shape while using the other hand to grasp and feel another stimulus, and noticed whether the two objects were identical in shape. If the objects were different, the object in the right hand was dropped and another object was grasped and compared to the object in the left hand. If the objects matched, participants released both stimuli and immediately started the cycle over, beginning with the other hand than in the previous cycle.

During the prescan training sessions on the grasping tasks, subjects observed a sample performance of the tasks and then were blindfolded and performed each task without having their hands covered from view from the experimenters who closely monitored and coached their performance. During task performance in the fMRI sessions, subjects were also observed by the experimenters to ensure that the movements were consistent with those during training. The goal of the training and observation during scanning was to ensure that the movements in the two tasks were as comparable as possible. During the prescan training sessions on the cutaneous tasks, subjects observed a sample performance of the tasks and performed each task as experimenters closely monitored the perfor- mance to ensure that there was no finger, hand, limb, or body movement.

The fMRI images were made with a wholebody MRI system operating at $81 \mathrm{mHz}$ (Gyrex, Elscint, Ltd., Israel). The subject's head was immobilized with a facial mask. A body coil was used for the radio-frequency transmission. The signal was received by a quadrature surface coil (US Asia Instruments, Inc., Highland Heights, OH). The dentate nuclei were functionally mapped with $\mathrm{T}_{2}{ }^{*}$ gradient-echo images (Drayer et al. 1986). To locate the dentate nucleus for functional scanning, each session was started by using several high-resolution T2-weighted spin-echo images in the transverse plane perpendicular to the brain stem to cover the entire cerebellum. Two or three of the resulting images always contain the dentate nucleus, which is readily identified in these images because it is relatively dark (as a result of its high-iron-content dentate, it has low signal intensity). On the basis of the location of the dentate in the latter images, we selected the functional scanning image plane (of 6-mm thickness) to pass through the middle of the dentate. Human dentate nucleus is $-7-11 \mathrm{~mm}$ in the direction parallel to the brain stem, but most of its mass is concentrated in the middle (thinning out at the upper and lower ends). Thus, image planes we selected for recording functional activation are likely to contain much more than $60 \%$ of the dentate. Typically, the $\mathrm{T}_{2}{ }^{*}$-weighted images were collected by a conventional gradient-echo sequence with three cycles of the Rest Control and each of two tasks. Twenty images were acquired for each task within each cycle ( $7.8 \mathrm{sec}$ per image). Imaging parameters were as follows: echo time, $40 \mathrm{msec}$; repetition time, $60 \mathrm{msec}$; and flip angle, $20^{\circ}$. Images were acquired with 256 complex pairs in the readout direction and 128 phase-encoding steps in a field of view $25.6 \times 25.6 \mathrm{~cm}^{2}$, with an in-plane spatial resolution of 1 by $2 \mathrm{~mm}^{2}$. The acquired $128 \times 256 \mathrm{fMRI}$ data were zero-filled to $256 \times 256$, then Fourier-transformed. Maps of functional activation were calculated by comparison of $T_{2}{ }^{*}$ weighted images acquired during the rest control with those acquired during tasks (Xiong et al. 1995).

Activation (Figs. 2 and 3, below) was defined by the combination of two criteria to afford a 0.05 level of statistical significance. First, a $t$-test was used to compare the resting baseline to task activation, and only pixels with a significant activation $(t>2.5)$ were included in the functional map. Second, regions with less than five contiguous acti-

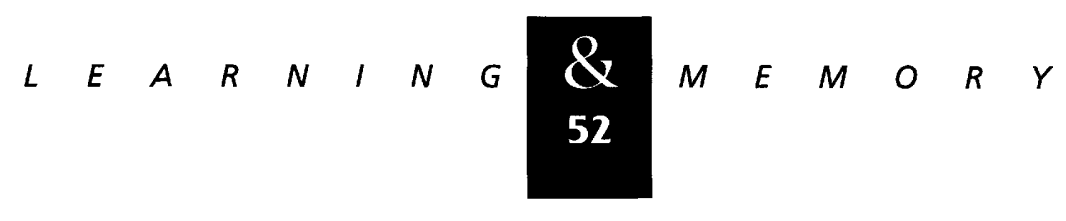


vated pixels were excluded. This cluster analysis method addresses the multiple comparison problem while maintaining reasonable sensitivity (for discussion, see Friston et al. 1994; Forman et al. 1995; Xiong et al. 1995). This strategy of using two thresholds (intensity and area) is based on the fact that neural activity tends to cluster in spatial location and that MRI noise tends to be spatially uncorrelated. The resulting functional map was laid over a $\mathrm{T}_{2}{ }^{*}$-weighted image to determine activation sites. The area of activation used for comparisons was the number of activated pixels within the dentate nuclei. Omnibus statistics and planned comparisons were made with separate ANOVAs and Newman-Keuls tests of subjects' activation area values and intensity values for left and right dentate nuclei.

\section{Results}

All four predictions of the hypothesis were confirmed. Figure 1 shows the statistically signifi- cant, group-averaged, task-induced functional MRI (black) for each task laid onto group-averaged anatomical MRI (gray). Figures 2 and 3 quantify in bar graphs the extent and intensity, respectively, of the statistically significant, group-averaged, task-induced activation detected for each task.

The dentate nuclei showed significant task-induced increases in blood flow in the CS condition. Thus, dentate nuclei are activated by purely sensory stimuli, confirming positron-emission tomography results showing cerebellar activation during hand vibration (Fox et al. 1985). This activation was equally strong in the left and right dentate but tended to be more extensive in the right dentate, probably reflecting the left cerebral dominance of our right-handed subjects. Known anatomical connections (Brodal 1978; Glickstein et al. 1985; Schmahmann and Pandya 1995) may enable cerebellar participation in such sensory processing.

When the same stimuli were presented under identical conditions, but a discrimination was required (CD), dentate nuclei were more than twice
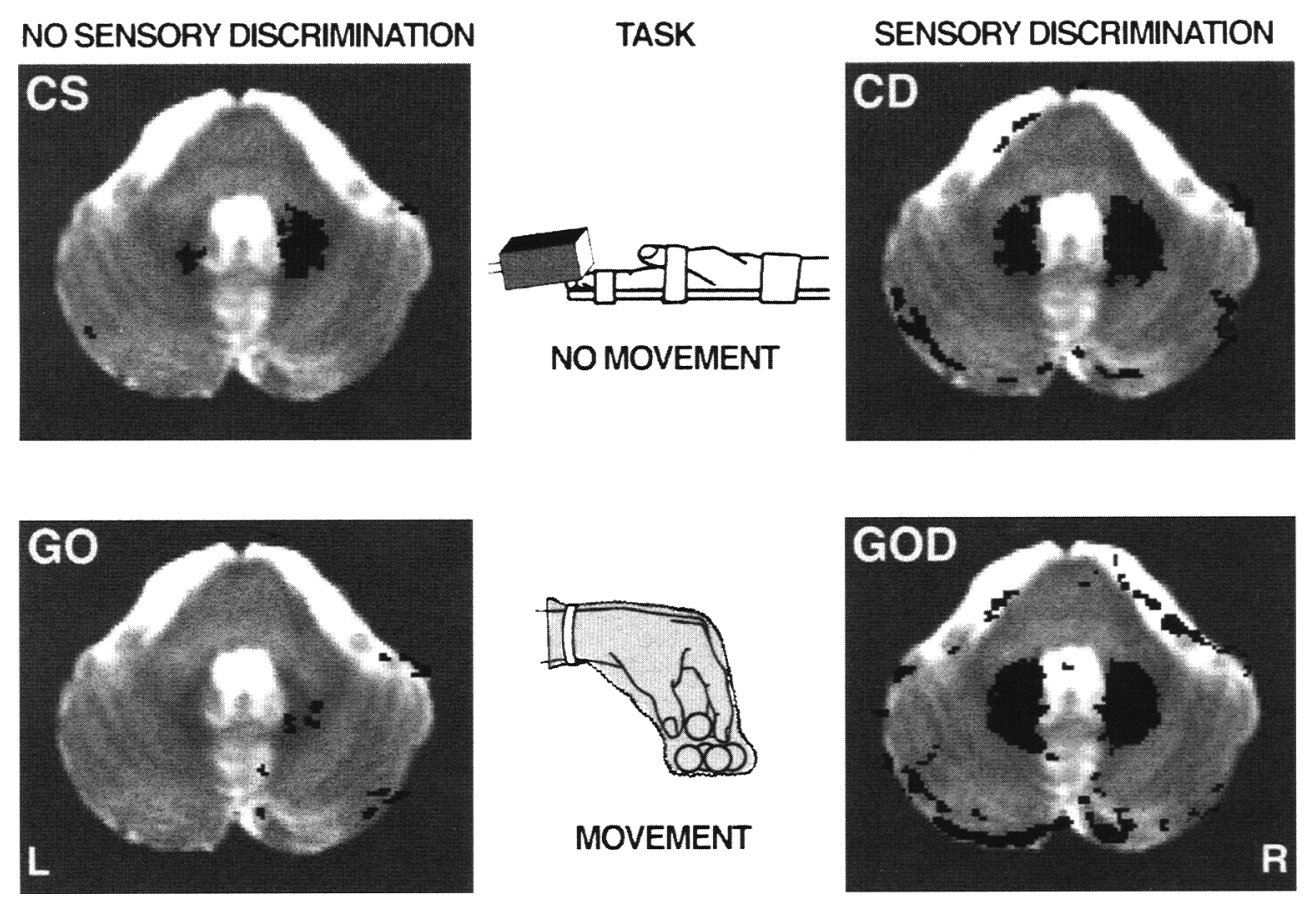

Figure 1: Functional MRI (black) laid onto anatomical MRI (gray) showing dentate activations for CS (top left), CD (top right), GO (bottom left), and GOD (bottom right) tasks. The dentate nuclei are the two dark crescent-shaped structures on either side of the cerebellar midline. Functional and anatomical images were coregistered for each task by performing rotation, translation, and scaling on each subject's images and then averaging images across subjects. A group t-test was performed on these images for each task comparing task-induced changes relative to rest. Activation was detected with a threshold defined by both a $t$ value of 2.5 and a cluster of five adjacent pixels. The detected activations are statistically significant to $P<0.05$ relative to the whole cerebellar plane sampled and shown in black scale ranging from a $t$ value of 2.5 to 10.0 .

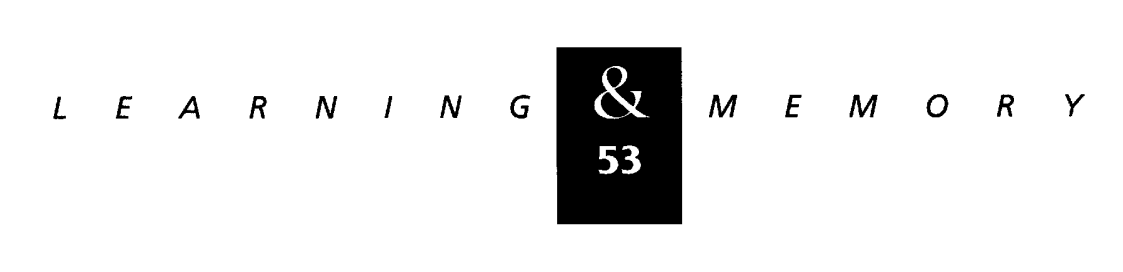




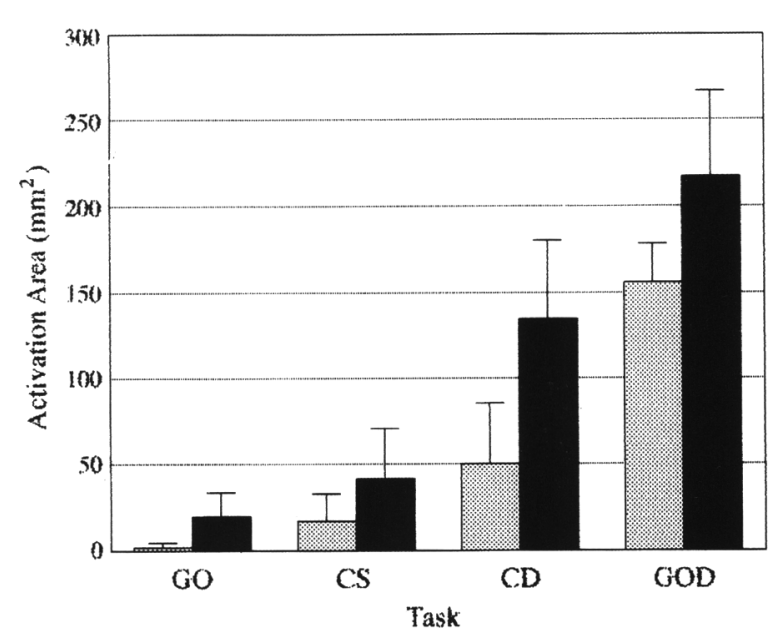

Figure 2: The activation area in the dentate nuclei for each task. (Shaded bar) Left dentate; (solid bar) right dentate. A group $t$-test was applied to each subject's data for each task compared with rest. Then, for each subject, activation foci were detected by selecting areas that had a $t$ value $>2.5$ and at least five adjacent pixels (corresponding in combination to $P<0.05$ ). The mean $( \pm$ S.E.M.) of these activated foci was calculated for each task across subjects.

as active $(P<0.05)$. This activation was bilateral, but stronger in the right dentate. The enhanced activity could reflect the anatomical connections between these cerebellar regions and the prefrontal cortex that supports working memory processes (Baddeley 1986; Middleton and Strick 1994; Goldman-Rakic 1995) that are possibly necessary for discrimination. The activations observed in these two cutaneous tasks are thus consistent with the hypothesis that the cerebellum is engaged during the acquisition and processing of sensory information and is even more strongly engaged during discrimination. It is not yet known what mechanism or pathway is responsible for this increase in activity with the requirement to make a discrimination; we hypothesize that this increase reflects an anticipatory influence of the cerebral cortex on the cerebellum (Morissette and Bower 1996).

We also compared cerebellar activation in a sensory discrimination task requiring rapid coordinated finger movements to a control task with similar movements, but no discrimination. The control task (GO) produced very slight, statistically insignificant activation. The slight activation likely reflects cutaneous stimulation of the fingers touching the stimuli. The lack of activation in the GO Task confirms that rapid coordinated, fine finger movements, in the absence of a sensory discrimination, do not engage the dentate nucleus. This response is equal to the slight dentate activity recorded in another fine motor behavior (Kim et al. 1994). In the latter study, the subject used visual guidance to reach to and grasp a peg, then raise and place it in the next small hole in linear array of such holes. The lack of dentate activation in such fine motor control tasks is opposite of what would be expected if existing theories of cerebellar motor control were correct and is evidence that the primary role of the cerebellum is not coordination of motor behavior for its own sake. The fact that active finegrained finger movements do not alone significantly activate the dentate nuclei indicates that even if during either cutaneous task subjects made finger movements too slight to be detected by the experimenters, those movements per se would not cause the significant activations. This conclusion is further assurance that the dentate is indeed specifically activated by the acquisition and discrimination of tactile sensory information.

By far the strongest activation occurred when subjects made a covert discrimination of object

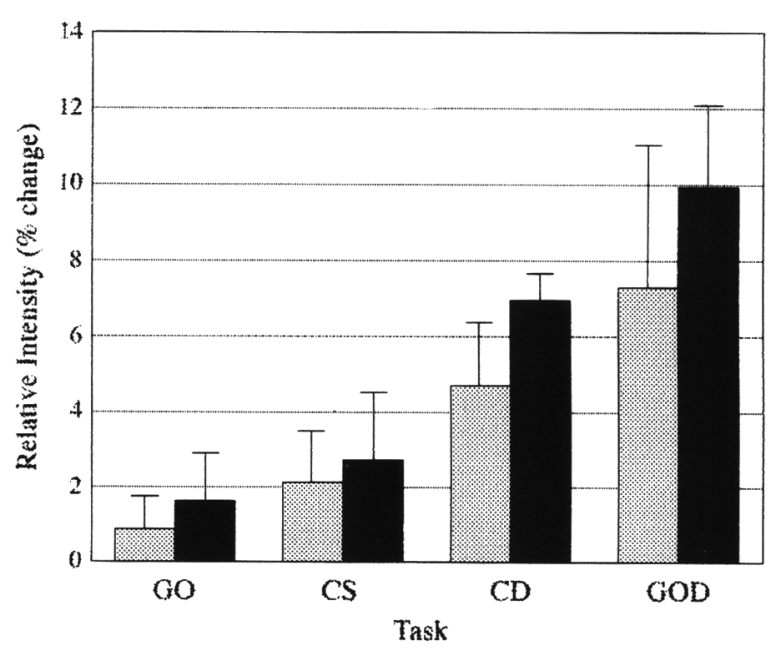

Figure 3: The intrinsic relative signal changes at the activation area in the dentate nuclei for each task. (Shaded bar) Left dentate; (solid bar) right dentate. A group $t$-test was applied to each subject's data for each task compared with rest. Then, for each subject, activation foci were detected by selecting areas that had a $t$ value $>2.5$ and at least five adjacent pixels (corresponding in combination to $P<0.05$ ). For each subject, the relative signal change was calculated in the abovethreshold activation areas by subtracting the average signal value during rest from that during task and dividing by the average signal value during rest. Mean relative signal change ( \pm S.E.M.) was calculated for each task across subjects.

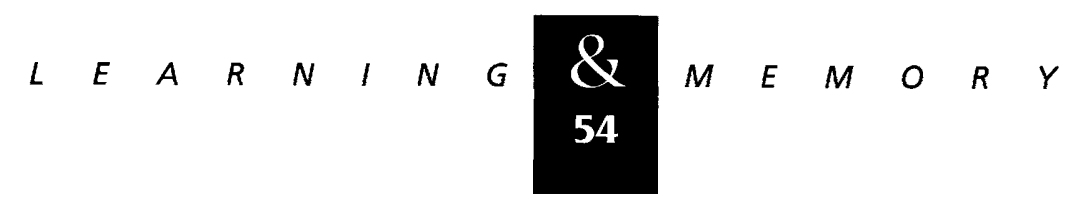


shape using their fingers (GOD). Again, right dentate was slightly more active than in the left. There was $>23$ times as much activation observed when a discrimination was required in the grasping task than when it was not, and this extreme contrast $(P<0.005)$ documents strong cerebellar support for sensory discrimination. These data are consistent with earlier position emission tomography (PET) experiments reporting posterior cerebellar activity during tactile exploration tasks (Seitz et al. 1991).

Taken together, these results rule out the conclusion that the greater dentate activity in the GOD Task simply reflects fine motor control in general. The GO Task, which requires similarly fine motor control, produces no significant dentate activation. Thus, fine movement control per se does not engage the dentate, in contrast to sensory stimulation per se. The massive increase in activity in the GOD Task relative to that in the GO Task is entirely out of proportion to whatever subtle differences exist between the two tasks' very similar finger movements. The primary difference in movementsthat GOD was performed at a slightly slower pacewould incorrectly predict a decrease in activation because motor performance rate and activation strength are positively correlated (Seitz et al. 1990; Sabatini et al. 1993). Thus, we are not claiming that the finger movements in GOD and GO were identical, just that both types of movements fall within the generally accepted category of "fine finger control" and that from the point of view of motor theories of cerebellum the enormous difference in activation would be completely unexpected.

There was also significant activation detected in the cortex of the lateral cerebellar hemispheres contained in the image plane (Fig. 1). Across the four conditions, activity in the lateral cerebellar cortex was positively correlated $(r=+0.91$, $P<0.02)$ with that in the dentate. The extent of statistically significant, task-induced, group-averaged activation detected in cerebellar cortex and dentate are quantified in the bar graph in Figure 4. The relationship between the dentate nucleus and cerebellar cortex is complex; nonetheless, this finding, although based on a small sample of cerebellar cortex, supports our hypothesis about the function of lateral hemispheres.

\section{Discussion}

In summary, our results consistently implicate the dentate nucleus of the human cerebellum in

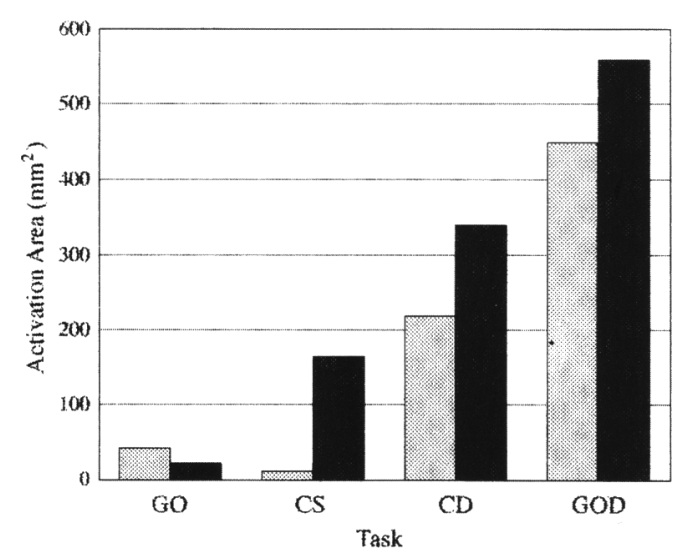

Figure 4: Bilateral activation in cerebellar cortex (shaded bar) and in dentate nuclei (solid bar) in the image plane sampled here. The extent of activation (in $\mathrm{mm}^{2}$ ) is plotted for each of the four tasks used in this study.

sensory acquisition and discrimination. Activation occurred during sensory stimulation when there were no accompanying overt finger movements or discrimination. Substantial finger movements, when not associated with tactile discrimination, did not induce significant activation. Dentate activation was greatly enhanced when a sensory discrimination was required, with or without overt finger movements. However, the strongest activation occurred when sensory discrimination was combined with finger movements.

Although these findings implicate the lateral cerebellum in sensory discrimination rather than movement per se, they do not identify its specific role. For example, it is possible to interpret the greater increase in dentate activity for the grasping discrimination task as simply being attributable to the increased complexity of this sensory processing task compared with the pure cutaneous discrimination (Gibson 1962; Clark and Horch 1986; Lederman and Klatsky 1987; Parsons and Shimojo 1987; Reed et al. 1996). Discrimination based on the active manipulation of objects presumably involves a greater variety and amount of information to be acquired and integrated over time and space, including information about multiple finger positions, the three-dimensional spatial arrangement of object surfaces constituting shape, and tactile information about specific surfaces. In contrast, the cutaneous discrimination task involved passively forming a representation of a uniform surface composed of few dimensions of information (i.e., coarseness). The additional processing required to

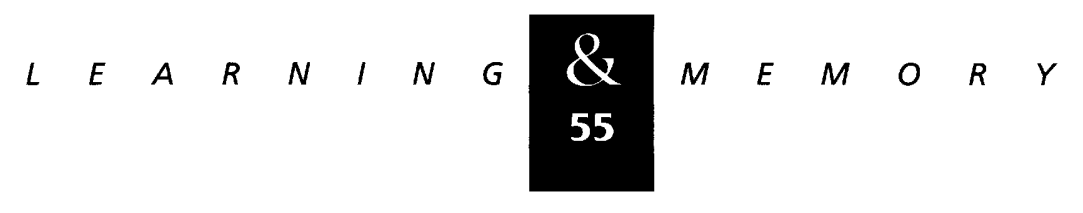


represent the more complex stimuli for the purpose of discrimination could be distributed among the somatosensory cortex, working memory areas in prefrontal cortex, and the cerebellum. As discussed earlier, there is appropriate connectivity for such distributed function. On the other hand, the interpretation of these differences in activation that is most in keeping with the hypothesis we set out to test is that the higher levels of cerebellar activation in the case of active manipulation reflect a direct role of the cerebellum in modulating the finger motor control system to reposition the tactile sensory surfaces of the fingers. This coordination may be based on the cerebellar analysis of the sense data actually being acquired and serves to assure that the highest quality information about object shape is being obtained in a coordinated fashion from all surfaces involved in the discrimination (Bower 1997a,b).

These findings are sharply contrary to the prevailing framework of cerebellar theory. Although space does not permit a full discussion of how one might comprehend or reconcile the contradictions, we will briefly describe below some of the more important issues involved. First, the data reported here are reliable. As noted in Results, the data in each separate condition are very similar to those of studies with other subjects, from other neuroimaging modalities, and by other research groups working with different experimental hypotheses and theoretical orientations. In addition, we have replicated the results using different subjects and a different imaging modality, PET, to measure activity throughout the whole cerebellum and cerebral cortex (L.M. Parsons, J.M. Bower, J. Xiong, M. Saenz, and P.T. Fox, unpubl.). Second, as described in the introduction, these tasks were designed to specifically test a new cerebellar theory previously developed over several years (Bower and Kassel 1990; Bower 1997a,b). The theory was not generated after the fact to fit the data. Third, as noted in Results, the data are internally consistent, each finding directly implied by our explicit hypothesis of cerebellar function. Fourth, motor control functions cannot explain the dentate and cerebellar cortex activation in response either to nonmotor sensory tasks or to the requirement to a make a sensory discrimination (see Results). Fifth, as described below, our hypotheses and findings are not inconsistent with principal observations of cerebellar research such as the behavioral effects of lesions; the possible role of cerebellum in associative learning; neurophysiological studies of non- human cerebellum in awake, behaving subjects; cerebellar participation in nonmotor, perceptual, and cognitive tasks; and the role of the cerebellar flocculus in regulation of the vestibulo-ocular reflex.

The hypotheses and findings reported here are not inconsistent with principal effects of cerebellar damage on behavior. For example, cerebellar deficits in voluntary movement such as incoordination and ataxia may result from disruption of the sensory data on which the motor system depends, rather than from disruption of cerebellar computations of smooth motor performance per se. Specifically, such deficits could be attributable to disruption of the known influence by the anterior cerebellum on the gamma motor system that is responsible for providing essential data to the motor system during motor control. Likewise, the decreased rate at which cerebellar patients perform motor tasks can be explained by the present hypothesis because longer computing time would be expected to process poorly controlled sensory data (for studies on patients' movement times, see Hallett et al. 1991; Wild and Dichgans 1992; Jahanshahi et al. 1993; Haggard et al. 1994; Muller and Dichgans 1994; Wessel et al. 1994; B. Wild and J. Dichgans, unpubl.). The same explanation accounts for the known increase in performance latencies for sensory-related tasks (Ivry and Keele 1989; Keele and Ivry 1990; Wessel et al. 1994; Tachibana et al. 1995). Furthermore, whereas many discriminations and behaviors can be completed in spite of the slowdown owing to the hypothesized lack of good sensory data, in tasks involving timing, any slowdown in processing time would be expected to interfere with task completion. For this reason, this hypothesis predicts that cerebellar dysfunction should have a noticeable effect on tasks involving timing, for which there is ample evidence (Ivry and Keele 1989; Keele and Ivry 1990; Lundyekman et al. 1991). Finally, the hypothesis would accommodate the suggested cerebellar involvement in autism (Courchesne et al. 1994; also see Filapek 1995) because a dysfunctional cerebellum would yield inconsistent or variable control of sensory data acquisition and would result in the reported difficulties autistic children have in dealing with sensory stimulation.

Our findings are also not incompatible with the data concerning some cerebellar involvement in associative learning. For example, several researchers have reported that specific cerebellar lesions interfere with the ability of rabbits to associ-

$$
\text { n...... }
$$


ate conditioned stimuli with eye blinks (Thompson and Krupa 1994). Although these experiments are controversial (Welsh and Harvey 1992; Bloedel and Bracha 1995; Logan and Grafton 1995), the point here is that none of those studies have taken into account the possibility that the sensory data on which the classical conditioning is dependent has changed. If cerebellar lesions do disrupt the structure of the sensory data, then one would also expect a disruption in conditioned responses, although it then becomes impossible to draw conclusions about the location of the "memory trace" from these experiments.

In addition, our results are not inconsistent with the many rigorous neurophysiological studies of awake behaving animals producing data interpreted as indicating that the cerebellum is involved in the planning and control of motor behavior. This view of the cerebellum is supported by recent trends in the types of tasks required of primates during cerebellar recording sessions. Specifically, early experiments of this type required monkeys to make large-scale controlled movements of the arms while the hands were in contact with a manipulandum (Thach 1970; Gilbert and Thach 1977; Harvey et al. 1977; Bauswein et al. 1983; Houk and Gibson 1987; Dugas and Smith 1992). These studies generally did not consider the possibility that recorded cerebellar responses might be more related to the changing tactile contact of the hand with the manipulandum. Similar experiments and interpretations have also been applied to cats (Schwartz et al. 1987; Lou and Bloedel 1992; Apps et al. 1995). More recently, researchers, largely driven by the desire to produce more robust cerebellar responses (cf. Houk and Gibson 1987; van Kan et al. 1994), have been training monkeys to make fine movements of the fingers that are increasingly like sensory discrimination tasks (Houk and Gibson 1987; Dugas and Smith 1992; van Kan et al. 1994; Robinson 1995; Sinkjaer et al. 1995). Although these studies are still interpreted in the context of motor control and do clearly have a motor component, they also require considerably more sensory processing than the old tasks involving the smooth movement of bars. Unfortunately, these animal studies (as well as other neuroimaging studies) did not attempt to decouple the sensory and motor processing as we have done here. As a result, it is plausible that cerebellar activity in the latter studies was also owing to acquisition of proprioceptive or tactile information rather than to computation involving motor coordination per se.
Our data are also compatible with the cerebellum participation in many varieties of sensory-motor, sensory, higher perceptual, and higher cognitive tasks (for reviews, see Fiez 1996; Bower 1997a,b; Parsons and Fox 1997; Schmahmann 1997; for related discussion see Bower 1995; Leiner et al. 1995). If the function of the cerebellum is to support the control of the acquisition of sensory data in general, not just tactile information, then it follows that the cerebellum should be active during a range of behaviors, including motor, perceptual, and cognitive tasks, all of which entail the acquisition of sensory information.

Finally, these data and our hypothesis about the role of cerebellar hemispheres in the somatosensory system are perhaps most consistent with the well-established role of the cerebellum in eye movement control. By definition, all eye movement control is directly related to sensory data acquisition, as this is all the eyes do. The specific role played by the cerebellum in eye movement control is perhaps best understood in the floccular regions of the cerebellum involved in the regulation of the vestibulo-ocular reflex. This reflex specifically compensates for the dislocation of images on the retina as a result of body movement (Paulin et al. $1989 a, b)$. It is already known that the flocculus receives information signaling the occurrence of retinal slip during head movements (Ito et al. 1982) and is presumed to be involved in reducing the amount of retinal slip. Although the cerebellum effects this control through the extraoccular eye muscles (Ito et al. 1982), the computational objective of the cerebellar control is to reduce retinal slip, which is known to degrade the processing of visual information in the visual cortex (Westheimer and McKee 1975). The flocculus does not take part in the discrimination of visual objects, but it increases the efficiency of function of the visual system. The present hypothesis proposes a comparable involvement for the lateral cerebellar hemispheres in tactile data acquisition. Interestingly, the flocculus is one of the phylogentically oldest cerebellar regions, whereas the cerebellar hemispheres are the youngest (Larsell 1972).

In conclusion, the contribution of the cerebellum to sensory acquisition and discrimination must be investigated further to be clearly understood. Experiments in preparation have studied these same four tasks while neuroimaging the activity in the red and dentate nuclei (Y. Pu, J.-H. Gao, Y.J. Liu, M. Liotti, P.T. Fox, J.M. Bower, and L.M. Parsons, unpubl.) and while neuroimaging the whole

$$
\text { ……‥ }
$$


brain and whole cerebellum, using correlated activation to evaluate hypotheses about distributed function (L.M. Parsons, J.M. Bower, J. Xiong, M. Saenz, and P.T. Fox, unpubl.). More generally, as the variety of behavior in which the cerebellum is reported to participate increases, it becomes critical to disentangle the component elementary operations of each behavior and determine where and to what extent each operation is selectively supported within the cerebellum. The benefit of such a strategy is demonstrated in evidence now emerging for regional dissociation of function (for review, see, e.g., Parsons and Fox 1997). At the same time, a thorough understanding of cerebellar function will depend on developing a better understanding of the computational significance of cerebellar cortical and deep nuclear circuitry (see, e.g., De Schutter and Maex 1996; Jaeger et al. 1997). Finally, as our grasp of the principles of distributed computation deepens, hypotheses about cerebellar function are likely to have quite a different form and be supported by quite different arguments, than at present.

\section{Acknowledgments}

This work was supported by an EJLB Foundation grant and National Institute of Mental Health grants P20 DA52176-01 and MH/DA52145 (Human Brain Project), and MH/DA52145-04 (Human Brain Project) to J.M.B.

The publication costs of this article were defrayed in part by payment of page charges. This article must therefore be hereby marked "advertisement" in accordance with 18 USC section 1734 solely to indicate this fact.

\section{References}

Allen, G., R.B. Buxton, E.C. Wong, and E. Courchesne. 1997. Attentional activation of the cerebellum independent of motor involvement. Science 275: 1940-1943.

Apps, R., N.A. Hartell, and D.M. Armstrong. 1995. Step phase-related excitability changes in spino-olivocerebellar paths to the $\mathrm{c}(1)$ and $\mathrm{c}(3)$ zones in cat cerebellum. J. Physiol. (Lond.) 483: 687-702.

Asanuma, C., W.T. Thach, and E.G. Jones. 1983. Brain-stem and spinal projections of the deep cerebellar nuclei in the monkey, with observations on the brain-stem projections of the dorsal column nuclei. Brain Res. Rev. 5: 299-322.

Aumann, T.D., J.A. Rawson, D.I. Finkelstein, and M.K. Horne. 1994. Projections from the lateral and interposed cerebellar nuclei to the thalamus of the rat-a light and electron-microscopic study using single and double anterograde labeling. J. Comp. Neurol. 349: 165-181.

Baddeley, A. 1986. Working memory. Oxford University Press, New York, NY.
Bauswein, E., F.P. Kolb, B. Leimbeck, and F.J. Rubia. 1983 Simple and complex spike activity in cerebellar Purkinje cells during active and passive movements in the awake monkey. J. Physiol. (Lond.) 339: 379-394.

Bloedel, J.R. and T.M. Kelly. 1992. The dynamic selection hypothesis: A proposed function for cerebellar sagittal zones. In The cerebellum revisited (ed. R.R. Llinás and C. Sotelo), pp. 267-282. Springer-Verlag, New York, NY.

Bloedel, J.R. and V. Bracha. 1995. On the cerebellum, cutaneomuscular reflexes, movement control, and the elusive engrams of memory. Behav. Brain Res. 68: 1-44.

Bower, J.M. 1992. Is the cerebellum a motor control device? Behav. Brain Sci. 15: 714-715.

1995. The cerebellum as a sensory acquisition controller: Commentary on "The underestimated cerebellum" by Leiner et al. Hum. Brain Mapping 2: 255-256.

1997a. Is the cerebellum sensory for motor's sake, or motor for sensory's sake: The view from the whiskers of a rat? In Progress Brain Research (ed. C.I. de Zeeuw, P. Strata, and J. Voogd), pp. 483-516. Elsevier Science, Amsterdam, The Netherlands.

1997b. Control of sensory data acquisition. In The cerebellum and cognition (ed. J.D. Schmahmann). Academic Press, New York, NY.

Bower, J.M. and D.C. Woolston. 1983. Congruence of spatial organization of tactile projections to granule cell and Purkinje cell layers of cerebellar hemispheres of the albino rat: Vertical organization of cerebellar cortex. $J$. Neurophysiol. 49: 745-766.

Bower, J.M. and J. Kassell. 1990. Variability in tactile projection patterns to cerebellar folia crus-Ila of the Norway rat. J. Comp. Neurol. 302: 768-778.

Bracke-Tolkmitt, R., A. Linden, G.M. Canavan, B. Rockstroh, E. Scholz, K. Wessell, and H.C. Diener. 1989. The cerebellum contributes to mental skills. Behav. Neurosci. 103: 442-446.

Brodal, A. 1981. Neurological anatomy in relation to clinical medicine. Oxford University Press, New York, NY.

Brodal, P. 1978. The corticopontine projection in the rhesus monkey: Origin and principles of organization. Brain 101: 251-283.

Clark, F.J. and K.W. Horch. 1986. Kinethesia. In Handbook of perception and human performance: Vol. 1. sensory processes and perception (ed. K.R. Boff, L. Kaufman, and J.P. Thomas). Wiley, New York, NY.

Courchesne, E., J. Townsend, N.A. Akshoomoff, O. Saitoh, R. Yeung-Courchesne, A.J. Lincoln, H.E. James, R.H. Haas, L. Schreibman, and L. Lau. 1994. Impairment in shifting attention in autistic and cerebellar patients. Behav. Neurosci. 108: 848-865.

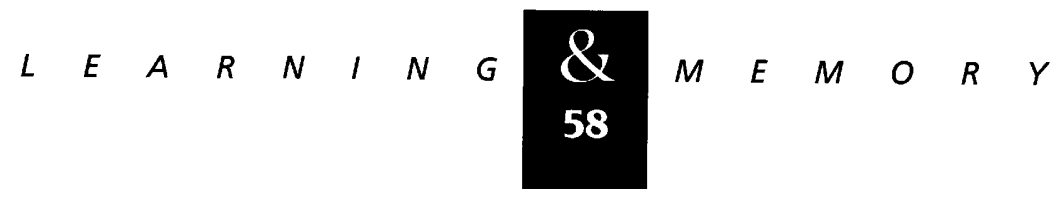


Daum, I. and H. Ackermann. 1995. Cerebellar contributions to cognition. Behav. Brain Res. 67: 201-210.

Dean, P. 1995. Modeling the role of the cerebellar fastigial nuclei in producing accurate saccades-The importance of burst timing. Neuroscience 68: 1059-1077.

De Schutter, E. and R. Maex. 1996. The cerebellum: Cortical processing and theory. Curr. Opin. Neurobiol. 6: 759-764.

Donga, R. and D. Dessem. 1993. An unrelayed projection of jaw-muscle spindle afferents to the cerebellum. Brain Res. 626: $347-350$.

Doyon, J. 1997. Skill learning. In The cerebellum and cognition (ed. J.D. Schmahmann). Academic Press, New York, NY.

Drayer, B., P. Burger, R. Darwin, S. Riederer, R. Herfkens, and G.A. Johnson. 1986. MRI of brain iron. Am. J. Roentgenol. 147: 103-110.

Dugas, C. and A.M. Smith. 1992. Responses of cerebellar Purkinje cells to slip of a hand-held object. J. Neurophysiol. 67: 483-495.

Ellerman, J.M., D. Flament, S.-G. Kim, Q.-G. Fu, H. Merkle, T.J. Ebner, and K. Ugurbil. 1994. Spatial patterns of functional activation of the cerebellum investigated using high-field (4-t) MRI. Magnetic Resonance Imaging Biomed. 7: 63-68.

Fiez, J.A. 1996. Cerebellar contributions to cognition. Neuron 116: 13-15.

Fiez, J. and M.E. Raichle. 1997. Linguistic processing. In The cerebellum and cognition (ed. J.D. Schmahmann). Academic Press, New York, NY.

Fiez, J.A., S.E. Petersen, M.K. Cheney, and M.E. Raichle. 1992. Impaired nonmotor learning and error-detection associated with cerebellar damage-a single case study. Brain 115: 155-178.

Filapek, P.A. 1995. Quantitative magnetic-resonance imaging in autism-the cerebellar vermis. Curr. Opin. Neurol. 8: 134-138.

Fox, P.T., M.E. Raichle, and W.T. Thach. 1985. Functional mapping of the human cerebellum with positron emission tomography. Proc. Natl. Acad. Sci. 82: 7462-7466.

Friston, K.J., P. Jezzard, and R. Turner. 1994. Analysis of functional MRI time-series. Hum. Brain Mapping 1: 153-171.

Ghelarducci, B. and L. Sebastiani. 1996. Contribution of the cerebellar vermis to cardiovascular control. J. Auton. Nerv. Syst. 56: 149-156.

Gibson, J.J. 1962. Observations on active touch. Psychol. Rev. 69: 477-491.
Gilbert, P.F.C. and W.T. Thach. 1977. Purkinje cell activity during motor learning. Brain Res. 128: 309-328.

Gilman, S., A.A.F. Sima, L. Junck, K.J. Kluin, R.A. Koeppe, M.E. Lohman, and R. Little. 1996. Spinocerebellar ataxia type- 1 with multiple system degeneration and glial cytoplasmic inclusions. Ann. Neurol. 39: 241-255.

Glickstein, M. 1993. Motor skills but not cognitive tasks. Trends Neurosci. 16: 450-451.

Glickstein, M., J. May, and B. Mercier. 1985. Corticopontine projection in the macaque: The distribution of labeled cortical cells after large injections of horseradish peroxidase in the pontine nuclei. J. Comp. Neurol. 235: 343-359.

Goldman-Rakic, P.S. 1995. Toward a circuit model of working memory and the guidance of voluntary motor action. In Models of information processing in the basal ganglia (ed. J.C. Houk, J.L. Davis, and D.G. Beiser), pp. 131-148.

Massachusetts Institute of Technology Press, Cambridge, MA.

Gonzalez, L., C. Shumway, J. Morissette, and J.M. Bower. 1993. Developmental plasticity in cerebellar tactile maps-fractured maps retain a fractured organization. J. Comp. Neurol. 332: 487-498.

Grafman, J., I. Litvan, S. Massaquoi, M. Stewart, A. Sirigu, and M. Hallett. 1992. Cognitive planning deficits in patients with cerebellar atrophy. Neurology 42: 1493-1496.

Haggard, P., J. Jenner, and A. Wing. 1994. Coordination of aimed movements in a case of unilateral cerebellar damage. Neuropsychologia 32: 827-846.

Hallett, M., A. Berardelli, J. Matheson, J. Rothwell, and C.D. Marsden. 1991. Physiological analysis of simple rapid movements in patients with cerebellar deficits. J. Neurol. Neurosurg. Psychiatry 54: 124-133.

Harvey, R.J., R. Porter, and J.A. Rawson. 1977. The natural discharges of Purkinje cells in paravermal regions of lobules $\mathrm{V}$ and VI of the monkey's cerebellum. J. Physiol. (Lond.) 271: 515-536.

Holmes, G. 1917. The symptoms of acute cerebellar injuries due to gunshot wounds. Brain 40: 461-535.

1939. The cerebellum of man. Brain 62: $1-30$.

Horak, F.B. and H.C. Diener. 1994. Cerebellar control of postural scaling and central set in stance. J. Neurophysiol. 72: 479-493.

Houk, J.C. and A.R. Gibson. 1987. Sensorimotor processing through the cerebellum. In New concepts in cerebellar neurobiology (ed. J.S. King), pp. 387-416. A.R. Liss, New York, NY.

Houk, J.C. and S.P. Wise. 1995. Distributed modular architectures linking basal ganglia, cerebellum, and cerebral-cortex-their role in planning and controlling action. Cereb. Cortex 5: 95-110. 


\section{Parsons et al.}

Ito, M. 1993. Movement and thought-identical control mechanisms by the cerebellum. Trends Neurosci.

16: $448-450$.

Ito, M., M. Sakurai, and P. Tongroach. 1982. Climbing fibre induced depression of both mossy fibre responsiveness and glutamate sensitivity of cerebellar Purkinje cells. J. Physiol. (Lond.) 324: 113-134.

Ivry, R.B. and S.W. Keele. 1989. Timing functions of the cerebellum. J. Cognit. Neurosci. 1: 136-152.

Jacquin, M.F. and H.P. Zeigler. 1983. Trigeminal orosensation and ingestive behavior in the rat. Behav. Neurosci. 97: 62-97.

Jaeger, D. and J.M. Bower. 1994. Prolonged responses in rat cerebellar Purkinje-cells following activation of the granule cell layer-An intracellular in-vitro and in-vivo investigation. Exp. Brain Res 100: 200-214.

Jaeger, D., E. De Schutter, and J.M. Bower. 1997. The role of synaptic and voltage-gated currents in the control of Purkinje cell spiking: A modeling study. J. Neurosci. 17: 91-106.

Jahanshahi, M., R.G. Brown, and C.D. Marsden. 1993. A comparative-study of simple and choice-reaction time in parkinsons, huntingtons and cerebellar disease. J. Neurol. Neurosurg. Psychiatry 56: 1169-1177.

Jueptner, M., M. Rijntjes, C. Weiller, H.H. Faiss, D. Timmann, S.P. Mueller, and H.C. Diener. 1995. Localization of a cerebellar timing process using PET. Neurology 45: $1540-1545$.

Kawato, M. and H. Gomi. 1992. A computational model of 4 regions of the cerebellum based on feedback-error learning. Biol. Cybern. 68: 95-103.

Keele, S.W. and R. Ivry. 1990. Does the cerebellum provide a common computation for diverse tasks-a timing hypothesis. Ann. N.Y. Acad. Sci. 608: 179-211.

Keifer, J. and J.C. Houk. 1994. Motor function of the cerebellorubrospinal system. Physiol. Rev. 74: 509-542.

Kim, S.-G., K. Ugurbil, and P.L. Strick. 1994. Activation of a cerebellar output nucleus during cognitive processing. Science 265: 949-951.

Larsell, O. 1972. The comparitive anatomy and histology of the cerebellum from monotremes through apes. University of Minnesotta Press, Minneapolis, MN.

Lederman, S.J. and R.L. Klatsky. 1987. Hand movements: A window into haptic object recognition. Cognit. Psychol. 19: $342-368$.

Leiner, H.C., A.L. Leiner, and R.S. Dow. 1995. The underestimated cerebellum. Hum. Brain Map. 2: 244-254.

Llinás, R. 1984. Functional significance of the basic cerebellar circuit in motor coordination. In Cerebellar functions (ed. J.R. Bloedel), pp. 171-185. Springer-Verlag, New York, NY.

Logan, C.G. and S.T. Grafton. 1995. Functional anatomy of human eyeblink conditioning determined with regional cerebral glucose metabolism and positron-emission tomography. Proc. Natl. Acad. Sci. 92: $7500-7504$

Lou, J.-S. and J.R. Bloedel. 1992. Responses of sagittally aligned Purkinje cells during perturbed locomotion: Relation of climbing fiber activation to simple spike modulation. J. Neurophysiol. 68: 1820-1833.

Lundyekman, L., R. Ivry, S. Keele, and M. Woollacott. 1991. Timing and force control deficits in clumsy children. J. Cognitive Neurosci. 3: 367-376.

Marr, D. 1969. A theory of cerebellar cortex. J. Physiol. (Lond.) 202: 437-471.

Martin, P. and M. Albers. 1995. Cerebellum and schizophrenia-a selective review. Schizophr. Bull. 21: $241-250$.

Middleton, F.A. and P.L. Strick. 1994. Anatomical evidence for cerebellar and basal ganglia involvement in higher cognitive function. Science 266: 458-461.

Morissette, J. and J.M. Bower. 1996. The contribution of somatosensory cortex to responses in the rat cerebellar granule cell layer following peripheral tactile stimulation. Exp. Brain Res. 109: 240-250.

Muller, F. and J. Dichgans. 1994. Dyscoordination of pinch and lift forces during grasp in patients with cerebellar lesions. Exp. Brain Res. 101: 485-492.

Naito, Y., A. Newman, W.S. Lee, K. Beykirch, and V. Honrubia. 1995. Projections of the individual vestibular end-organs in the brain-stem of the squirrel-monkey. Hear. Res. 87: 141-155.

Nisimaru, N. and S. Katayama. 1995. Projection of cardiovascular afferents to the lateral nodulus-uvula of the cerebellum in rabbits. Neurosci. Res. 21:343-350.

Ojakangas, C.L. and T.J. Ebner. 1992. Purkinje-cell complex and simple spike changes during a voluntary arm movement learning-task in the monkey. J. Neurophysiol. 68: 2222-2236.

1994. Purkinje-cell complex spike activity during voluntary motor learning-relationship to kinematics. J. Neurophysiol. 72: 2617-2630.

Parsons, L.M. and S. Shimojo. 1987. Perceived spatial organization of cutaneous patterns on surfaces of the human body in various positions. J. Exp. Psychol. 13: 488-504.

Parsons, L.M. and P.T. Fox. 1997. Sensory and cognitive functions. In The cerebellum and cognition (ed. J.D. Schmahmann), pp. 255-271. Academic Press, New York, NY.

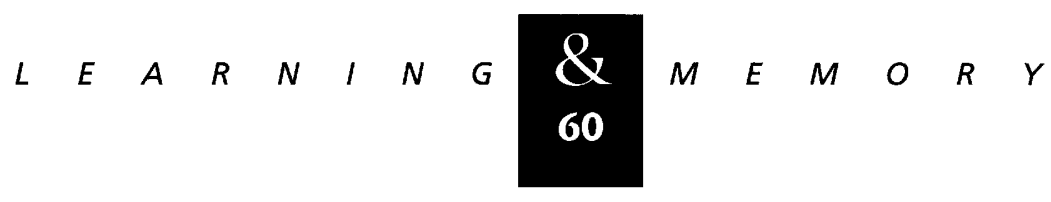


Parsons, L.M., P.T. Fox, J.H. Downs, T. Glass, T.B. Hirsch, C.C. Martin, P.A. Jerabek, and J.L. Lancaster. 1995. Use of implicit motor imagery for visual shape discrimination as revealed by PET. Nature 375: 54-59.

Paulin, M.G. 1993. The role of the cerebellum in motor control and perception. Brain Behav. Evol. 41: 39-50.

Paulin, M.G., M.E. Nelson, and J.M. Bower. 1989a. Dynamics of compensatory eye movement control: An optimal estimation analysis of the vestibulo-ocular reflex. Int. J. Neural Syst. 1: 23-29.

1989b. Neural control of sensory acquisition: The vestibulo-ocular reflex. In Advances in neural information processing systems (ed. D. Touretzky), Vol. 1, pp. 410-418. Morgan Kaufmann, San Mateo, CA.

Petersen, S.E., P.T. Fox, M.I. Posner, M. Mintun, and M.E. Raichle. 1989. Positron emission tomographic studies of the processing of single words. J. Cognit. Neurosci. 1: 153-170.

Reed, C.L., R.J. Caselli, and M.J. Farah. 1996. Tactile agnosia: Underlying impairment and implications for normal tactile object recognition. Brain 119: 875-888.

Robinson, F.R. 1995. Role of the cerebellum in movement control and adaptation. Curr. Opin. Neurobiol. 5: 755-762.

Rubia, F.J. and J.B. Phelps. 1970. Responses of the cerebellar cortex to cutaneous and visceral afferents. Pflügers Arch. 314: 68-85.

Sabatini, U., F. Chollet, O. Rascol, P. Celsis, A. Rascol, G.L. Lenzi, and J.-P. Marc-Vergnes. 1993. Effect of side and rate of stimulation on cerebral blood flow changes in motor areas during finger movements in humans. J. Cereb. Blood Flow Metab. 13: 639-645.

Sasaki, K., J.M. Bower, and R. Llinás. 1989. Multiple purkinje-cell recording in rodent cerebellar cortex. Eur. J. Neurosci. 1: 572-586.

Schmahmann, J.D. 1996. From movement to thought: Structural and functional correlates of the cerebellar contribution to cognitive processing. Hum. Brain Mapping 4: 174-198.

Schmahmann, J.D., ed. 1997. The cerebellum and cognition. Academic Press, New York, NY.

Schmahmann, J.D. and D.N. Pandya. 1995. Prefrontal cortex projections to the basilar pons: Implications for the cerebellar contribution to higher function. Neurosci. Lett. 199: $175-178$.

Schwartz, A.B., T.J. Ebner, and J.R. Bloedel. 1987. Responses of interposed and dentate neurons to perturbations of the locomotor cycle. Exp. Brain Res. 67: 323-338.

Seitz, R.J, P.E. Roland, C. Bohm, T. Greitz, and S. Stone-Elander. 1990. Motor learning in man. NeuroReport 1: $57-60$.

\begin{abstract}
1991. Somatosensory discrimination of shape-tactile exploration and cerebral activation. Eur. J. Neurosci. 3: 481-492.

Shulman, G.L., M. Corbetta, R.L. Buckner, J.A. Fiez, F.M. Miezin, M.E. Raichle, and S.E. Petersen. 1997. Common blood flow changes across visual tasks: I. Increases in subcortical structures and cerebellum, but not in non-visual cortex. J. Cognit. Neurosci. (in press).
\end{abstract}

Sinkjaer, T., L. Miller, T. Andersen, and J.C. Houk. 1995. Synaptic linkages between red nucleus cells and limb muscles during a multijoint motor task. Exp. Brain Res. 102: $546-550$.

Snider, R.S. and A. Stowell. 1944. Receiving areas of the tactile, auditory, and visual systems in the cerebellum. J. Neurophysiol. 7: 331-357.

Strick, P.L. 1983. The influence of motor preparation on the response of cerebellar neurons to limb displacements. J. Neurosci. 3: 2007-2020.

Subramony, S.H. 1994. Degenerative ataxias. Curr. Opin. Neurol. 7: 316-322.

Tachibana, H., K. Aragane, and M. Sugita. 1995. Event-related potentials in patients with cerebellar degeneration-electrophysiological evidence for cognitive impairment. Cognit. Brain Res. 2: 173-180.

Thach, W.T. 1970. Discharge of cerebellar neurons related to two maintained postures and two prompt movements: Purkinje cell output and input. J. Neurophysiol. 33: 537-547.

1978. Correlation of neural discharge with pattern and force of muscular activity, joint position, and direction of intended next movement in motor cortex and cerebellum. $J$. Neurophysiol. 41: 654-676.

1997. Cerebellum, motor learning and thinking in man: Pet studies. Behav. Brain Sci. (in press).

Thach, W.T., H.P. Goodkin, and J.G. Keating. 1992. The cerebellum and the adaptive coordination of movement. Annu. Rev. Neurosci. 15: 403-442.

Thompson, R.F. and D.J. Krupa. 1994. The organization of memory traces in the mammalian brain. Annu. Rev. Neurosci. 17: 519-549.

van Kan, P.L.E., J.C. Houk, and A.R. Gibson. 1993. Output organization of intermediate cerebellum in monkey. J. Neurophysiol. 69: 57-73.

van Kan, P.L.E., K.M. Horn, and A.R. Gibson. 1994. The importance of hand use to discharge of interpositus neurons of the monkey. J. Physiol. (Lond.) 480: 171-190.

Welker, W. 1987. Spatial organization of somatosensory projections to granule cell cerebellar cortex: Functional and connectional implications of fractured somatotropy. In New 


\section{Parsons et al.}

concepts in cerebellar neurobiology (ed. J.S. King), pp. 239-280. Liss, New York, NY.

Welsh, J.P. and J.A. Harvey. 1992. The role of the cerebellum in voluntary and reflexive movements. In The cerebellum revisited (ed. R.R. Llinás and C. Cotelo), pp. 301-334. Springer-Verlag, New York, NY.

Welsh, J.P., E.J. Lang, I. Sugihara, and R. Llinás. 1995. Dynamic organization of motor control within the olivocerebellar system. Nature 374: 453-457.

Wessel, K., R. Verleger, D. Nazarenus, P. Vieregge, and D. Kompf. 1994. Movement-related cortical potentials preceding sequential and goal-directed finger and arm movements in patients with cerebellar atrophy. Electroencephalogr. Clin. Neurophysiol. 92: 331-341.

Westheimer, G. and S.P. McKee. 1975. Visual acuity in the presence of retinal-image motion. J. Opt. Soc. Am. 65: $847-850$.

Xiong, J., J.-H. Gao, J.L. Lancaster, and P.T. Fox. 1995. Clustered pixels analysis for functional MRI activation studies of the human brain. Hum. Brain Mapping 3: 287-301.

Yang, B.Y. and D.J. Weisz. 1992. An auditory conditioned-stimulus modulates unconditioned stimulus elicited neuronal-activity in the cerebellar anterior interpositus and dentate nuclei during nictitating-membrane response conditioning in rabbits. Behav. Neurosci. 106: 889-899.

Received February 14, 1997; accepted in revised form May 2, 1997. 


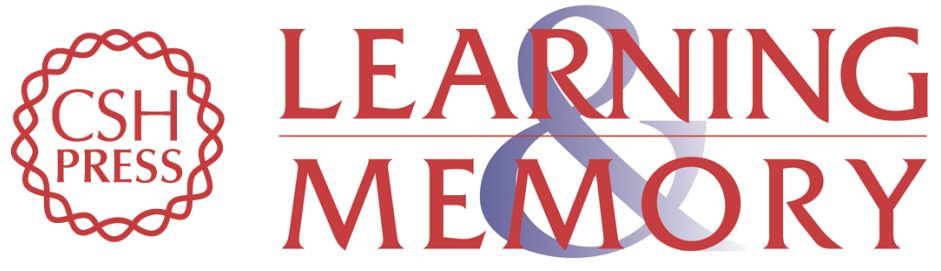

\section{Lateral cerebellar hemispheres actively support sensory acquisition and discrimination rather than motor control.}

L M Parsons, J M Bower, J H Gao, et al.

Learn. Mem. 1997, 4:

Access the most recent version at doi:10.1101//m.4.1.49

References This article cites 97 articles, 11 of which can be accessed free at:

http://learnmem.cshlp.org/content/4/1/49.full.html\#ref-list-1

License

Email Alerting Receive free email alerts when new articles cite this article - sign up in the box at the Service top right corner of the article or click here. 